\title{
Robust Regression Estimation Methods and Intercept Bias: A Capital Asset Pricing Model Application
}

\author{
James B. McDonald \\ Brigham Young University, USA \\ Richard A. Michelfelder \\ Rutgers University, USA \\ Panayiotis Theodossiou \\ Rutgers University, USA \\ Cyprus University of Technology, Cyprus
}

Robust estimation techniques based on symmetric probability distributions are often substituted for OLS to obtain efficient regression parameters with thick-tail distributed data. The empirical, simulation and theoretical results in this paper show that with skewed distributed data, symmetric robust estimation techniques produce biased regression intercepts. This paper evaluates robust methods in estimating the capital asset pricing model and shows skewed stock returns data used with symmetric robust estimation techniques produce biased alphas. The results support the recommendation that robust estimation using the skewed generalized $\mathrm{T}$ family of distributions may be used to obtain more efficient and unbiased estimates with skewness. (JEL: G12, C13, C14, C15).

Keywords: CAPM; quasi-maximum likelihood estimator; robust estimator; skewed generalized $\mathrm{T}$

\footnotetext{
* The authors thank the Special Issue Editor, Prof. Richard Taffler and an anonymous reviewer for being helpful and providing refining comments. We also thank the participants at the 13th Multinational Finance Society Annual Conference in Edinburgh for helpful comments on earlier versions of this paper, and Brad Larsen, currently a graduate student at MIT, for his highly diligent research assistance. We would also like to recognize the Whitcomb Center for Research in Financial Services for providing research support through the use of the WRDS system.
}

(Multinational Finance Journal, 2009, vol. 13, no. 3/4, pp. 293-321)

(C) Multinational Finance Society, a nonprofit corporation. All rights reserved.

DOI: $10.17578 / 13-3 / 4-6$ 


\section{Introduction}

Robust regression methods usually address violations of assumptions of ordinary least squares (OLS) to produce efficient estimators. When researchers substitute OLS for another estimator to improve estimator efficiency, the robust estimators they use in place of OLS may introduce bias to the intercept. Secondly, the OLS slope is inefficient when the error distribution is thick-tailed or skewed, whereas robust methods usually address thick tails. Specifying a log-likelihood function with a non-normal symmetric density to address OLS inefficiency introduces bias to the intercept if the errors are skewed as well as thick-tailed.

This paper shows that robust estimators using the skewed generalized T (SGT), a recently developed five-parameter distribution that accommodates both skewness and thicktails, provides an efficient and unbiased estimator of the intercept and slope when the error distribution is skewed and / or thick-tailed. The SGT nests the normal distribution and many commonly used distributions in robust estimation.

The issue of a biased intercept is important in areas as estimating the capital asset pricing model (CAPM) since the intercept, known as Jensen's alpha ((Jensen (1968)) is used to evaluate the return performance of stocks and mutual funds, stock valuation, and for predicting the market returns on an asset. Generally, the problem of a biased intercept is important in any analysis where the intercept has an important interpretation or prediction estimates of the dependent variable are generated by the regression model. For example, biased intercepts will systematically over- or under-state forecasts.

It is a generally accepted finding that stock returns are usually nonnormally distributed, and their empirical distributions often exhibit both kurtosis and skewness; e.g., Mandelbrot (1963), Fama (1965), Fama, Fisher, Jensen and Roll (1969), Fielitz and Smith (1972), Francis (1975) and McDonald and Nelson (1989). A major issue investigated in this paper is the effect of skewness in stock returns on the estimation of the CAPM, a two-parameter linear model that involves regressing excess stock returns on excess stock market returns.

Estimating CAPM regressions with OLS produces unbiased and efficient parameter estimates when the stock returns and associated regression errors are normally distributed. Blume (1968) shows that non-normal stock returns generate non-normal residuals in CAPM estimation. When the error distribution is thick-tailed and/or skewed, OLS produces inefficient estimates. The latter necessitates the use of robust, quasi-maximum likelihood, or partially adaptive estimation techniques. 
Some robust estimation techniques commonly used include the least absolute deviation (LAD) estimator which minimizes the sum of the absolute value of the regression errors or, more generally, $L_{k}$ estimators which minimize the sum of absolute values of the regression errors raised to the power $k$ for fixed, but unrestricted value of $k$. Still more general robust estimators include the $L_{k}$ estimators where the data endogenize the selection of the value of the parameter $k$ or M-estimators which minimize a general function of the errors over the parameter values. LAD and $L_{k}$ (with $k$ predetermined or determined by the data) are both special cases of M-estimation. See Hampel (1974), Huber (1981), Koenker (1982), Koenker and Basset (1978), and Koenker and Basset (1982) for a more thorough discussion of these estimation techniques.

Another type of robust estimator involves the choice of alternatives to the normal density for regression estimation such as generalized $\mathrm{T}$ (GT), generalized error (GED), student's T, and Laplace, which is the LAD. There are many robust estimation methods and we do not attempt to develop an exhaustive discussion of all of them in this paper. They can be placed into two categories, one that is based on outlier-resistant methods in choosing regression parameter estimates, and the other the choice of probability density function (pdf) and parameters for specifying the likelihood function. These types may overlap. Boyer, McDonald, and Newey (2003) differentiate robust or outlier-resistant estimators into re-weighted least squares or least median squares, and partially adaptive estimators. Partially adaptive estimation procedures can be viewed as being quasi-maximum likelihood estimators (QMLE) because they maximize a log-likelihood function corresponding to an approximating error distribution over both regression and distributional parameters. In other words they maximize a likelihood function by choosing values for the regression parameters and the distribution parameters of the regression residuals. Hinich and Talwar (1975), Chan and Lakonishok (1992), Yohai and Zamar (1997), Martin and Simin (2003) also have developed outlier resistant methods for efficiency improvement.

The above robust techniques address efficiency due to kurtosis, but do not account for skewness. If skewness is present, the estimated CAPM intercept will be biased downwards in positively skewed data and upwards in negatively skewed data. The size of the bias is directly related to the extent of skewness and kurtosis. The CAPM intercept, or Jensen's alpha, is frequently used as a measure of stock portfolio performance. A biased alpha can lead to erroneous decisions on stock 
valuation, portfolio selection, and mutual fund investment evaluation. Moreover, stocks with biased alphas can lead to biased and inefficient portfolios; see Frankfurter, Phillips, and Seagle (1974). Inefficient betas (CAPM slope) can also lead to large errors in estimating the cost of common equity capital as shown in McDonald, Michelfelder, and Theodossiou (2009).

The above problem is important to address in any regression if the intercept has a meaningful interpretation or if the regression model is used in forecasting. A solution to the problem of both parameter inefficiency and intercept bias in regression is to use "flexible" probability distribution functions (pdf's), that is, those that accommodate both kurtosis and skewness. Such flexible pdf's include the SGT of Theodossiou (1998), the skewed generalized error (SGED) of Theodossiou (2001), the inverse hyperbolic sine (IHS) of Johnson (1949) and the exponential generalized beta of the second kind (EGB2) of McDonald and Xu (1995). Hansen, McDonald, and Theodossiou (2007) include some additional discussion of these distributions and applications.

This paper provides theoretical, empirical, and simulation verifications of the intercept bias and shows how to address the bias problem. It also evaluates many well-known robust estimation methods using nine error distributions nested within the SGT that have various restrictions in accommodating thick-tails and skewness. The SGT nesting provides us with an integrated framework for making comparisons of the techniques such as LAD, trimmed regression quantile, $L_{k}$, and $\mathrm{M}$ estimators and reaching conclusions on the relative efficiency of the regression parameters. The paper employees the entire universe of publicly traded stocks in the U.S. which had at least four years of usable data over the period 1995 to 2004.

The next section of the paper discusses the CAPM estimation, the properties of the SGT, and the bias in the intercept. Section III reviews the empirical results. Section IV discusses the simulation results involving CAPM regressions where normal, thick-tailed, and skewed error pdf's are used to simulate regression errors. Section V concludes the paper.

\section{SGT-Capm Estimation}

The estimation of CAPM's parameters is accomplished by fitting the following regression equation to each stock's returns data: 


$$
r_{i, t}=\alpha_{i}+\beta_{i} r_{M, t}+\varepsilon_{i, t},
$$

$$
\text { for } i=1,2, \ldots, N \text { and } t=1,2, \ldots, T_{i} \text {, }
$$

where $r_{i, t}=R_{i, t}-R_{f, t}$ and $r_{M, t}=R_{M, t}-R_{f, t}$ are excess returns from the risk free rate $R_{f, t}$ for stock $i$ and the market, $\alpha_{i}$ and $\beta_{i}$ are the alpha and beta for the stock, $\varepsilon_{i, t}$ is a regression error term for each individual stock's return generating process having zero mean and constant variance, $T_{i}$ is the sample size for the stock, and $N$ is the number of stocks. Note in the above CAPM specification the value for each stock's alpha implied by the theory is zero since $R_{f, t}$ is subtracted from both sides of the equations and otherwise $R_{f, t}$ is the theoretical intercept. As such, the above equation is often used to test the validity of the CAPM model for stocks and other assets as well as to assess the performance of stocks and mutual funds. A positive alpha would indicate that the stock or mutual fund had superior returns relative to risk and vice versa.

Estimates for the alpha and beta of each stock are obtained from the maximization of the sample log-likelihood function

$$
\max _{\theta_{i}} l\left(\theta_{i}\right)=\sum_{t=1}^{T_{i}} \ln f\left(r_{i, t} \mid r_{M, t}, \theta_{i}\right)
$$

where $f$ is the hypothesized probability density function for $r_{i, t}$ and $\theta_{i}=$ $\left[\alpha_{i}, \beta_{i}, \ldots\right]$ is a parameter vector for the alpha, beta and other distributional parameters. As in other studies, we use the non-centered SGT log-likelihood specification rather than the centered specification because it does not require the existence of the first and second moments and it is easier to estimate. See Theodossiou (1998) for additional estimation details for the SGT.

The non-centered SGT specification for stock's $i$ returns is

$$
\begin{aligned}
& f\left(r_{i, t} \mid r_{M, t}\right)=.5 k_{i}\left(\frac{n_{i}+1}{k_{i}}\right)^{-\left(\frac{1}{k_{i}}\right)} B\left(\frac{n_{i}}{k_{i}}, \frac{1}{k_{i}}\right)^{-1} \varphi_{i}^{-1} \\
& \left(1+\frac{\left|u_{i, t}\right|^{k_{i}}}{\left(\left(n_{i}+1\right) / k_{i}\right)\left(1+\operatorname{sign}\left(u_{i, t}\right) \lambda_{i}\right)^{k_{i}} \varphi_{i}^{k_{i}}}\right)^{-\left(\frac{n_{i}+1}{k_{i}}\right)}
\end{aligned}
$$


and

$$
u_{i, t}=r_{i, t}-\left(m_{i}+\beta_{i} r_{M, t}\right)
$$

where $u_{i, t}$ is a deviation of $r_{i, t}$ from its conditional mode $m_{i}+\beta_{i} r_{M, t}, \varphi_{i}$ is a scaling constant related to the standard deviation, when it exists, $B(\cdot)$ is the beta function, sign is the sign function taking the value of -1 for negative values of $u_{i, t}$ and 1 for positive values of $u_{i, t}, \lambda_{i}$ is a skewness parameter obeying the constraint $-1<\lambda_{i}<1$, and $k_{i}$ and $n_{i}$ are positive kurtosis parameters.

The parameter $k_{i}$ controls mainly the shape of the conditional density around the mode of $r_{i, t}$. Specifically, values of $k_{i}$ below two $\left(k_{\mathrm{i}}<2\right)$ result in density functions that are leptokurtic relative to the normal distribution (i.e., peaked around the mode) and values of $k_{i}$ greater than two $\left(k_{i}>2\right)$ result in density functions that are platykurtic relative to the normal distribution. As $k_{i}$ grows larger, the SGT density function approaches that of the uniform distribution. The parameter $n_{i}$ controls mainly the tails of the density. As $n_{i}$ gets smaller the tails of the SGT become fatter and as $n_{i}$ gets infinitely large, the SGT approaches the SGED and for $k=2$ the normal distribution. The standardized skewness and kurtosis values for the SGT can be obtained using equations (9) and (10) with feasible combinations as depicted in Hansen, McDonald, Theodossiou (2007) which covers a substantial portion of the area skewness $(-\infty, \infty)$, and $(1.8, \infty)$.

The non-centered SGT is defined for any value of $n_{i}>0$ and can be used in the estimation of the parameters $m_{i}$ and $\beta_{i}$ regardless of the existence of the first and second moments of the distribution. Note that the moments of the SGT exist up to the value $n_{i}$; see McDonald and Newey (1988) and Theodossiou (1998).

When $n_{i}>1$, the conditional expected value of $r_{i, t}$ is equal to

$$
\begin{gathered}
E\left(r_{i, t} \mid r_{M, t}\right)=\alpha_{i}+\beta_{i} r_{M, t}+E\left(\varepsilon_{i, t}\right)=\alpha_{i}+\beta_{i} r_{M, t} \\
=m_{i}+\beta_{i} r_{M, t}+E\left(u_{i, t}\right)=m_{i}+\beta_{i} r_{M, t}+\rho_{i} \varphi_{i}
\end{gathered}
$$

where

$$
\rho_{i}=2 \lambda_{i} B\left(\frac{n_{i}}{k_{i}}, \frac{1}{k_{i}}\right)^{-1}\left(\frac{n_{i}+1}{k_{i}}\right)^{\frac{1}{k_{i}}} B\left(\frac{n_{i}-1}{k_{i}}, \frac{2}{k_{i}}\right)
$$


Thus, the regression intercept of equation (1) is equal to

$$
\alpha_{i}=m_{i}+\rho_{i} \varphi_{i}
$$

When $n_{i}>2$, the conditional variance of returns exists and is equal to

$$
\sigma_{i}^{2}=\operatorname{var}\left(r_{i, t} \mid r_{M, t}\right)=\left(\gamma_{i}-\rho_{i}^{2}\right) \varphi_{i}^{2}
$$

where

$$
\gamma_{i}=\left(1+3 \lambda_{i}^{2}\right) B\left(\frac{n_{i}}{k_{i}}, \frac{1}{k_{i}}\right)^{-1}\left(\frac{n_{i}+1}{k_{i}}\right)^{\frac{2}{k_{i}}} B\left(\frac{n_{i}-2}{k_{i}}, \frac{3}{k_{i}}\right) .
$$

Equations (7) and (8) are necessary to compute the intercept and variance of a regression model when a non-centered density likelihood specification is used. Equation (7) can be rewritten as

$$
\alpha_{i}-m_{i}=\rho_{i} \varphi_{i}
$$

This equation provides the adjustment factor for the intercept when the non-centered SGT log-likelihood specification is used. Note that in the case of: a) negatively skewed SGT, $\lambda_{i}<0$ and $\rho_{i}<0$, b) symmetric SGT, $\lambda_{i}=0$ and $\rho_{i}=0$ and c) positively skewed SGT, $\lambda_{i}>0$ and $\rho_{i}>0$. The latter adjustment factor will be negative for negatively skewed returns and positive for positively skewed returns.

The skewness of $r_{i, t}$, for $n_{i}>3$, is

$$
\begin{gathered}
S K_{i}=\frac{m_{3, i}}{\sigma_{i}^{3}}=\frac{A_{3, i}-3 \gamma_{i} \rho_{i}+2 \rho_{i}^{3}}{\left(\gamma_{i}-\rho_{i}^{2}\right)^{3 / 2}} \\
\text { where } A_{3, i}=4 \lambda_{i}\left(1+\lambda_{i}^{2}\right) B\left(\frac{n_{i}}{k_{i}}, \frac{1}{k_{i}}\right)^{-1}\left(\frac{n_{i}+1}{k_{i}}\right)^{\frac{3}{k_{i}}} B\left(\frac{n_{i}-3}{k_{i}}, \frac{4}{k_{i}}\right) .
\end{gathered}
$$

The kurtosis of $r_{i, t}$, for $n_{i}>4$, is

$$
K U_{i}=\frac{m_{4, i}}{\sigma_{i}^{4}}=\frac{A_{4, i}-4 A_{3, i} \rho_{i}+6 \gamma_{i} \rho_{i}^{2}-3 \rho_{i}^{4}}{\left(\gamma_{i}-\rho_{i}^{2}\right)^{2}}
$$




$$
\text { where } A_{4, i}=\left(1+10 \lambda_{i}^{2}+5 \lambda_{i}^{4}\right) B\left(\frac{n_{i}}{k_{i}}, \frac{1}{k_{i}}\right)^{-1}\left(\frac{n_{i}+1}{k_{i}}\right)^{\frac{4}{k_{i}}} B\left(\frac{n_{i}-4}{k_{i}}, \frac{5}{k_{i}}\right)
$$

see the Appendix A for the derivations of the moments, skewness and kurtosis equations.

The SGT nests several well known pdf's as special cases, such as the GT, skewed T (ST), student's T, Cauchy, SGED, generalized error distribution (GED) and Laplace. Moreover, the SGT (with restrictions) log-likelihood specification yields the $L_{k}$ estimator, MAD (or LAD) estimator, and trimmed regression quantile estimator as special cases. See the Appendix B for more details.

\section{Sampling and Estimation}

We considered the population of all 11,001 common stocks in the University of Chicago's Center for Research in Security Prices (CRSP) database that were publicly traded between January 1, 1995 and December 31, 2004 on the NYSE, the AMEX, and the NASDAQ. Any stock was removed that did not have at least four years of data $(1,000$ trading-day returns) during the 10 year period. The time series observations of rates of return for individual stocks range between 1,000 and 2,519 observations. We avoided survival bias by not removing stocks that were de-listed or stopped trading due to liquidation from bankruptcy, dropped from trading on the exchange, merged, or exchanged for other stock. The resulting universe of 6,502 stocks were used in the analysis.

Stock returns $\left(R_{i, t}\right)$ are daily holding period rates of return for each stock obtained from the CRSP database. The risk-free rate of return $\left(R_{f, t}\right)$ is the daily return on the one-month US Treasury Bill that compounds to the monthly return for a specific month. The stock market return $\left(R_{m, t}\right)$ from the CRSP database is the value-weighted daily return on all of the stocks in the CRSP database. The daily excess stock market return $\left\{r_{m, t}\right.$ or $\left.\left(R_{m, t}-R_{f, t}\right)\right\}$, the independent variable in the regressions, is from the Fama and French files of the CRSP database.

All 6,502 stocks were used to compute regression estimates of alphas and betas using OLS, LAD, SLAD, GED, SGED, student's T, ST, GT, and SGT. We used the OLS estimates to initially analyze the pdf characteristics of the residuals as OLS is commonly used by 


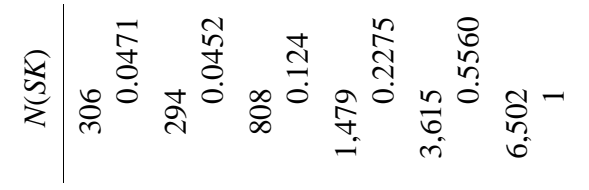

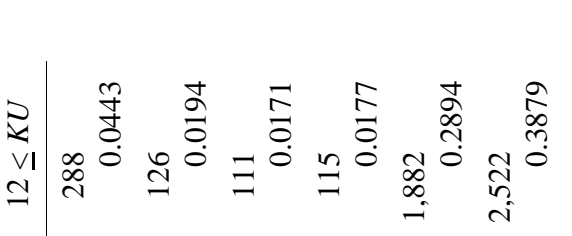

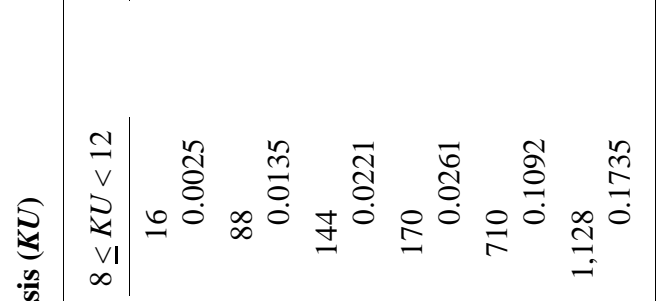

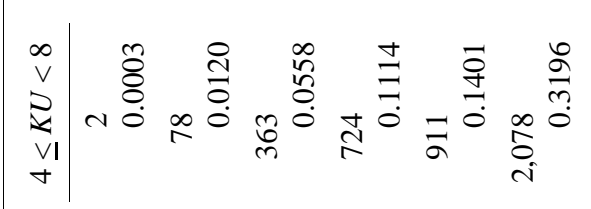

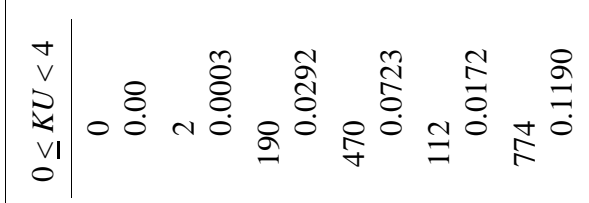

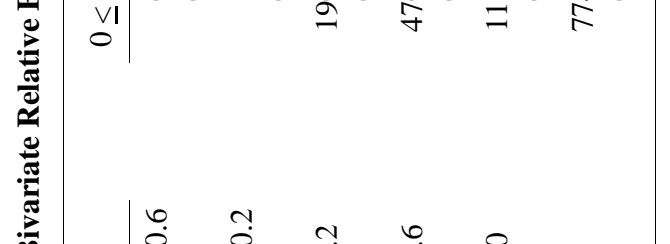

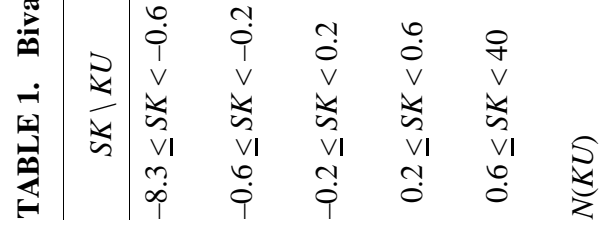

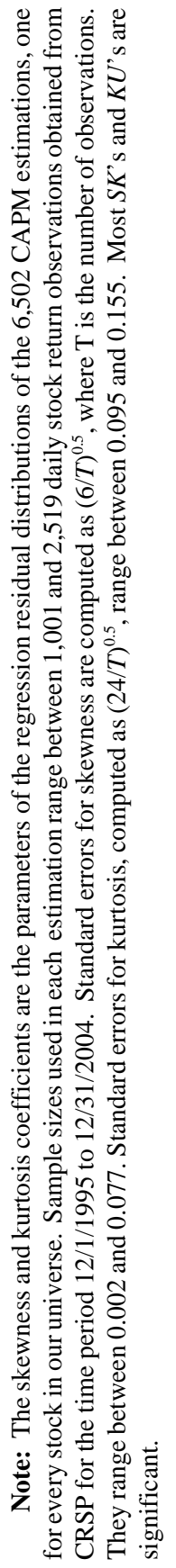


practitioners and many researchers of the CAPM.

A bivariate relative frequency table for the OLS regressions residuals skewness is presented on table 1 . The bottom row shows that $88 \%$ (5,728 stocks) of all regression residuals have standardized kurtosis greater than 4. Specifically, about 32\% (2,078 stocks) have kurtosis between 4 and 8,17\% (1,128 stocks) between 8 and 12 and $39 \%$ (2,522 stocks) greater than 12 . These results strongly support the hypothesis that the distribution of CAPM residuals are leptokurtic relative to the normal distribution. The last column of the table presents the results for the standardized skewness. According to the table, $88 \%$ $(5,694$ stocks) of the CAPM's skewness values are outside the -0.2 to 0.2 range, which roughly constitutes the confidence interval at the $5 \%$ level of significance (see table 1 notes for standard errors). Of these, about $9 \%$ (600 stocks) are less than -0.2 and 79\% (5,094 stocks) are greater than 0.2 , implying that the overwhelming majority of CAPM residuals are significantly positively skewed.

Interestingly the bivariate results of table 1 show that only about $3 \%$ (190 stocks) are approximately normally distributed as they exhibit skewness in the range of -0.2 to 0.2 and kurtosis in the range of 0 to 4 . Thus, about $97 \%$ (6,312 stocks) of CAPM residuals exhibit skewness and / or kurtosis. The results depict a positive relation between absolute skewness and kurtosis. In conclusion, the results of table 1 establish that robust estimation methods are required to obtain more efficient estimates of the CAPM's parameters as the majority of the CAPM regression residuals have both skewed and thick-tailed distributions.

We developed a frequency table of the Jarque-Bera (JB) statistic for the regressions residuals. The JB statistic tests the null hypothesis that the pdf of the regression residuals are normal. It performs a joint test for skewness and excess kurtosis and is $\chi^{2}$ distributed with two degrees of freedom. The results reject the null hypothesis of normality for all of the stocks' regression residuals. The JB table is available upon request.

Table 2 presents the frequency distribution of the betas estimated with the SGT. It shows that about $80 \%$ of the betas range from 0 to 1.5 . This is a reasonable range for the majority of beta levels. The associated estimated intercepts, although not shown but discussed below, are adjusted for the bias due to the structure of the SGT.

The first column of table 3 presents the frequency $N\left(\lambda_{i}\right)$ and relative frequency $P\left(\lambda_{i}\right)$ of the skewness parameter $\lambda_{i}$, for all 6,502 CAPM regressions, estimated using the SGT likelihood specification. Observe that about $21 \%(1,391$ stocks $)$ of the $\lambda_{i}$ 's are negative and about $79 \%$ 
TABLE 2. Beta Frequency Distribution: SGT Model

\begin{tabular}{|c|c|c|c|}
\hline \multicolumn{2}{|c|}{ Range } & $\begin{array}{l}\text { Number } \\
\text { of Stocks }\end{array}$ & $\begin{array}{l}\text { Fraction of } \\
\text { Total Sample }\end{array}$ \\
\hline-0.25 & 0.00 & 972 & 0.1495 \\
\hline 0.00 & 0.25 & 1,927 & 0.2964 \\
\hline 0.25 & 0.50 & 785 & 0.1207 \\
\hline 0.50 & 0.75 & 1,070 & 0.1646 \\
\hline 0.75 & 1.00 & 774 & 0.1190 \\
\hline 1.00 & 1.25 & 397 & 0.0611 \\
\hline 1.25 & 1.50 & 230 & 0.0354 \\
\hline 1.50 & 1.75 & 135 & 0.0208 \\
\hline 1.75 & 2.00 & 98 & 0.0151 \\
\hline 2.00 & 2.25 & 72 & 0.0111 \\
\hline \multicolumn{2}{|c|}{ Above 2.25} & 42 & 0.0065 \\
\hline \multicolumn{2}{|l|}{ Total } & 6,502 & 1 \\
\hline
\end{tabular}

Note: The beta is the estimated slope for the CAPM model. These results were estimated with 6,502 SGT regression estimations of the CAPM. Sample sizes used in the estimations range between 1,001 and 2,519 daily stock return observations obtained from CRSP for the time period $12 / 1 / 1995$ to $12 / 31 / 2004$. The average beta is 0.4763 .

$(5,111$ stocks) are positive. The last two columns give the number and fraction of $\lambda$ 's in each class interval that are statistically significant at the $5 \%$ and $1 \%$ levels, respectively. The t-values for the estimated $\lambda_{i}$ are based on robust standard errors. Notice that as we move away from zero, the fraction of statistically significant $\lambda$ 's in each class interval increases. The bottom row shows that about $50 \%$ (3,242 stocks) of the CAPM residuals exhibit significant positive or significant negative skewness. Of these, $21 \%$ (680 stocks) of the regression residuals exhibit negative skewness and 79\% (2,562 stocks) exhibit positive skewness. For the overall sample, the percentage of stocks with significant negative and significant positive skewness in CAPM residuals are respectively $10 \%(=680 / 6,502)$ and $39 \%(=2,562 / 6,502)$.

We developed frequency tables of the SGT kurtosis parameters, $n_{i}$ and $k_{\mathrm{i}}$ (not presented for brevity and available upon request). The $n_{i}$ parameter determines the thickness of the pdf's tails. Lower (higher) values of $n_{i}$ reflects thicker (thinner) tails for the SGT. About $51 \%$ $\left(3,316\right.$ stocks) of the $n_{i}$ 's range between 2 and 10 and are substantially lower than the normal pdf value of $n_{i}=30$. Less than $1 \%$ (52 stocks) of the estimated $n_{i}$ 's are less than one. Therefore the majority of the residuals have thick tailed pdf's, thereby driving the need for robustefficient estimators. 
TABLE 3. Relative Frequency of the Skewness Parameter $\lambda$

\begin{tabular}{|c|c|c|c|}
\hline Ranges & $\begin{array}{l}N(\lambda) / \\
P(\lambda)\end{array}$ & $\begin{array}{c}\text { Significant at the } \\
5 \% \text { level }\end{array}$ & $1 \%$ level \\
\hline$\lambda<-0.05$ & $\begin{array}{l}170 \\
0.0261\end{array}$ & $\begin{array}{l}126 \\
0.7412\end{array}$ & $\begin{array}{l}122 \\
0.7176\end{array}$ \\
\hline$-0.050 \leq \lambda<-0.025$ & $\begin{array}{l}377 \\
0.0580\end{array}$ & $\begin{array}{l}234 \\
0.6207\end{array}$ & $\begin{array}{l}221 \\
0.5862\end{array}$ \\
\hline$-0.025 \leq \lambda<0.000$ & $\begin{array}{l}844 \\
0.1298\end{array}$ & $\begin{array}{l}320 \\
0.3791\end{array}$ & $\begin{array}{l}285 \\
0.3377\end{array}$ \\
\hline $0.000 \leq \lambda<0.025$ & $\begin{array}{r}1,263 \\
0.1942\end{array}$ & $\begin{array}{l}229 \\
0.1813\end{array}$ & $\begin{array}{r}212 \\
0.1679\end{array}$ \\
\hline $0.025 \leq \lambda<0.050$ & $\begin{array}{r}1,247 \\
0.1918\end{array}$ & $\begin{array}{l}186 \\
0.1492\end{array}$ & $\begin{array}{l}111 \\
0.0890\end{array}$ \\
\hline $0.050 \leq \lambda<0.075$ & $\begin{array}{l}986 \\
0.1516\end{array}$ & $\begin{array}{l}603 \\
0.6116\end{array}$ & $\begin{array}{l}217 \\
0.2201\end{array}$ \\
\hline $0.075 \leq \lambda<0.100$ & $\begin{array}{l}708 \\
0.1089\end{array}$ & $\begin{array}{r}647 \\
0.9138\end{array}$ & $\begin{array}{l}502 \\
0.7090\end{array}$ \\
\hline $0.100 \leq \lambda<0.125$ & $\begin{array}{l}408 \\
0.0627\end{array}$ & $\begin{array}{l}400 \\
0.9804\end{array}$ & $\begin{array}{l}381 \\
0.9338\end{array}$ \\
\hline $0.125 \leq \lambda<0.150$ & $\begin{array}{l}261 \\
0.0401\end{array}$ & $\begin{array}{r}261 \\
1\end{array}$ & $\begin{array}{l}259 \\
0.9923\end{array}$ \\
\hline $0.150 \leq \lambda$ & $\begin{array}{l}238 \\
0.0366\end{array}$ & $\begin{array}{l}236 \\
0.9916\end{array}$ & $\begin{array}{l}236 \\
0.9916\end{array}$ \\
\hline $\begin{array}{l}\text { Total } \\
\text { Fraction of Total }\end{array}$ & $\begin{array}{r}6,502 \\
1\end{array}$ & $\begin{array}{r}3,242 \\
0.4986\end{array}$ & $\begin{array}{r}2,546 \\
0.3916\end{array}$ \\
\hline
\end{tabular}

Note: The skewness parameter, $\lambda$, for the SGT regression residuals distributions, is estimated from 6,502 CAPM SGT regressions. Sample sizes used in the estimations range between 1,001 and 2,519 daily stock return observations obtained from CRSP for the time period $12 / 1 / 1995$ to $12 / 31 / 2004$. The t-values used for significance tests are based on robust standard errors.

The $k_{\mathrm{i}}$ parameter determines the degree of leptokurtosis (platykurtosis). The value of $k_{\mathrm{i}}$ for OLS and the normal pdf is 2 . Values of $k_{\mathrm{i}}$ less than (greater than) 2 reflects leptokurtic (platykurtic) pdf's. We find that $k_{\mathrm{i}}$ is less than 1.75 for over $85 \%$ (5,527 stocks) of all residuals. The mean of $k_{\mathrm{i}}$ is 1.12 for all 6,502 SGT regressions. This is further evidence of mainly thick-tailed and peaked residual pdf's.

Note that to obtain the correct $\alpha_{i}$ value for the regression intercept, the quantity $\rho_{i} \varphi_{i}$ has to be added to the estimated mode intercept $m_{i}$, i.e., $\alpha_{i}=m_{i}+\rho_{i} \varphi_{i}$; see equation 7. Note, however, that the latter equation is only defined for values of $n_{i}>1$. The first column of table 4 presents the frequency $N\left(\rho_{i} \varphi_{i}\right)$ and relative frequency $P\left(\rho_{i} \varphi_{i}\right)$ of the intercept adjustment factor $\rho_{i} \varphi_{i}$, due to skewness, for 6,450 SGT- estimated 
TABLE 4. Relative Significance of Non-Centered SGT Adjustment Factor $\rho_{i} \varphi_{i}$

\begin{tabular}{|c|c|c|c|}
\hline$\rho_{i} \varphi_{i}$ & $\begin{array}{l}N\left(\rho_{i} \varphi_{i}\right) / \\
P\left(\rho_{i} \varphi_{i}\right)\end{array}$ & $\begin{array}{c}\text { Significant at the } \\
5 \% \text { level }\end{array}$ & $1 \%$ level \\
\hline$\rho_{i} \varphi_{i}<-0.5$ & $\begin{array}{l}118 \\
0.0181\end{array}$ & $\begin{array}{l}87 \\
0.7373\end{array}$ & $\begin{array}{l}86 \\
0.7288\end{array}$ \\
\hline$-0.50 \leq \rho_{i} \varphi_{i}<-0.25$ & $\begin{array}{l}275 \\
0.0423\end{array}$ & $\begin{array}{l}176 \\
0.6400\end{array}$ & $\begin{array}{l}167 \\
0.6073\end{array}$ \\
\hline$-0.25 \leq \rho_{i} \varphi_{i}<0.00$ & $\begin{array}{l}973 \\
0.1496\end{array}$ & $\begin{array}{l}404 \\
\quad 0.4152\end{array}$ & $\begin{array}{l}365 \\
0.3751\end{array}$ \\
\hline $0.00 \leq \rho_{i} \varphi_{i}<0.25$ & $\begin{array}{r}2,987 \\
0.4631\end{array}$ & $\begin{array}{l}813 \\
0.2722\end{array}$ & $\begin{array}{l}497 \\
0.1664\end{array}$ \\
\hline $0.25 \leq \rho_{i} \varphi_{i}<0.50$ & $\begin{array}{l}910 \\
0.1400\end{array}$ & $\begin{array}{l}630 \\
0.6923\end{array}$ & $\begin{array}{l}425 \\
0.4670\end{array}$ \\
\hline $0.50 \leq \rho_{i} \varphi_{i}<0.75$ & $\begin{array}{l}482 \\
0.0741\end{array}$ & $\begin{array}{l}430 \\
\quad 0.8921\end{array}$ & $\begin{array}{l}350 \\
0.7261\end{array}$ \\
\hline $0.75 \leq \rho_{i} \varphi_{i}<1.00$ & $\begin{array}{l}301 \\
\quad 0.0463\end{array}$ & $\begin{array}{l}290 \\
0.9635\end{array}$ & $\begin{array}{l}256 \\
0.8505\end{array}$ \\
\hline $1.00 \leq \rho_{i} \varphi_{i}<1.25$ & $\begin{array}{l}187 \\
0.0288\end{array}$ & $\begin{array}{l}179 \\
0.9572\end{array}$ & $\begin{array}{l}172 \\
0.9198\end{array}$ \\
\hline $1.25 \leq \rho_{i} \varphi_{i}<1.50$ & $\begin{array}{l}103 \\
0.0158\end{array}$ & $\begin{array}{l}103 \\
1.0000\end{array}$ & $\begin{array}{l}102 \\
0.9903\end{array}$ \\
\hline $1.50 \leq \rho_{i} \varphi_{i}$ & $\begin{array}{l}114 \\
0.0175\end{array}$ & $\begin{array}{r}114 \\
1\end{array}$ & $\begin{array}{r}114 \\
1\end{array}$ \\
\hline Total & 6,450 & 3,226 & 2,534 \\
\hline Fraction of Total & 1 & 0.5002 & 0.3929 \\
\hline
\end{tabular}

Note: The adjustment factor is estimated for each of the SGT residual distributions from 6,450 SGT CAPM regressions. The above table is based on a sample of 6,450 stocks, because 52 stocks had estimated values for $n_{i}<1$, thus $\rho_{i} \varphi_{i}$ was not defined. Sample sizes used in the estimations range between 1,001 and 2,519 daily stock return observations obtained from CRSP for the time period $12 / 1 / 1995$ to $12 / 31 / 2004$. For the computation of the significance frequency rates of the intercept adjustment factor we used the robust t-values of $\lambda$ 's.

CAPM regressions; i.e., 52 regressions yielded estimated values for $n_{i}$ $<1$. The last two columns give the number and fraction of $\rho_{i} \varphi_{i}$ 's in each class interval that are statistically significant at the 5\% and $1 \%$ levels, respectively. Notice that the results are quite analogous to those of table 3. This is a byproduct of the fact that the adjustment factor is driven mainly by the skewness parameter $\lambda_{i}$. Interestingly, the adjustment factor is in many instances greater than 0.5 or 50 basis points.

The estimated CAPM intercept bias due to skewness $b\left(\alpha_{i}\right)$ is computed as the difference between intercepts estimated using the SGT and GT (symmetric) likelihood specification, i.e., $b\left(\alpha_{i}\right)=\alpha_{S G T, i}-\alpha_{G T, i}$. 
TABLE 5. Relative Frequency of Intercept Bias Due to Skewness: SGT vs. GT

\begin{tabular}{|c|c|c|c|}
\hline$b\left(\alpha_{i}\right)=\alpha_{S G T, i}-\alpha_{G T, i}$ & $\begin{array}{l}N\left(b\left(\alpha_{i}\right)\right) / \\
P\left(b\left(\alpha_{i}\right)\right)\end{array}$ & $\begin{array}{c}\text { Significant at the } \\
5 \% \text { level }\end{array}$ & $1 \%$ level \\
\hline$b\left(\alpha_{i}\right)<-0.75$ & $\begin{array}{l}37 \\
0.0057\end{array}$ & $\begin{array}{l}29 \\
0.7838\end{array}$ & $\begin{array}{l}27 \\
0.7297\end{array}$ \\
\hline$-0.75 \leq b\left(\alpha_{i}\right)<-0.50$ & $\begin{array}{l}81 \\
0.0125\end{array}$ & $\begin{array}{l}69 \\
0.8519\end{array}$ & $\begin{array}{l}53 \\
0.6543\end{array}$ \\
\hline$-0.50 \leq b\left(\alpha_{i}\right)<-0.25$ & $\begin{array}{l}272 \\
0.0418\end{array}$ & $\begin{array}{l}211 \\
0.7757\end{array}$ & $\begin{array}{l}174 \\
0.6397\end{array}$ \\
\hline$-0.25 \leq b\left(\alpha_{i}\right)<0.00$ & $\begin{array}{r}1,035 \\
0.1592\end{array}$ & $\begin{array}{l}690 \\
0.6667\end{array}$ & $\begin{array}{l}591 \\
0.5710\end{array}$ \\
\hline $0.00 \leq b\left(\alpha_{i}\right)<0.25$ & $\begin{array}{r}4,243 \\
0.6526\end{array}$ & $\begin{array}{r}2,514 \\
0.5925\end{array}$ & $\begin{array}{r}1,720 \\
0.4054\end{array}$ \\
\hline $0.25 \leq b\left(\alpha_{i}\right)<0.50$ & $\begin{array}{l}659 \\
0.1014\end{array}$ & $\begin{array}{l}646 \\
0.9803\end{array}$ & $\begin{array}{l}585 \\
0.8877\end{array}$ \\
\hline $0.50 \leq b\left(\alpha_{i}\right)<0.75$ & $\begin{array}{l}141 \\
0.0217\end{array}$ & $\begin{array}{r}141 \\
1\end{array}$ & $\begin{array}{l}140 \\
0.9929\end{array}$ \\
\hline $0.75 \leq b\left(\alpha_{i}\right)$ & $\begin{array}{l}34 \\
0.0052\end{array}$ & $\begin{array}{r}34 \\
1\end{array}$ & $\begin{array}{r}34 \\
1\end{array}$ \\
\hline $\begin{array}{l}\text { Total } \\
\text { Fraction of Total }\end{array}$ & $\begin{array}{r}6,502 \\
1\end{array}$ & $\begin{array}{r}4,334 \\
0.6666\end{array}$ & $\begin{array}{r}3,324 \\
0.5112\end{array}$ \\
\hline
\end{tabular}

Note: The intercept bias is calculated as the difference between the SGT intercept and the intercept from its symmetric, restricted (skewness parameter $\lambda=0$ ) counterpart, the GT. Sample sizes used in the estimations range between 1,001 and 2,519 daily stock return observations obtained from CRSP for the time period 12/1/1995 to 12/31/2004. The test statistics for the bias significance are based on the log-likelihood ratio test statistic of the SGT (unrestricted) and GT (restricted) models. The ratio follows chi-square distribution with one degree of freedom.

Similarly, table 5 presents the relative frequency and significance results for the intercept bias $b\left(\alpha_{i}\right)$. The results show a significant difference in the intercepts of the skewed and symmetric likelihood specifications. In $67 \%(4,334$ stocks) of the cases, the bias is statistically significant at the $5 \%$ level and $51 \%(3,324$ stocks) are significant at the $1 \%$ level. Recall that in table $3,50 \%$ of the regressions' residuals distributions had significant skewness. The fraction of stocks that have significant bias with negative bias is $23 \%$ (999 stocks) and significant positive bias is $77 \%$ (3,335 stocks).

These results provide strong empirical support that when skewness is present in the data, the use of symmetric log-likelihood specifications or "symmetric type" robust estimation techniques will result in biased regression intercepts. This issue along with the issue of efficiency of 
various estimators is further investigated in the next section using simulations.

\section{Simulations and Estimation Performance}

We use Monte Carlo simulations to assess the effects of tail thickness and/or skewness of the error distribution on the relative efficiency of the various regression estimators of CAPM's intercept. In addition to OLS, LAD or Laplace, GED, student's T and GT -based estimators, used in prior studies, we consider the skewed specifications of Laplace (SLAD), GED, student T and GT estimators. These results extend those reported in Manski (1984) and McDonald and White (1993).

Specifically for the simulations, we use the CAPM model

$$
r_{t}=\alpha+\beta r_{M, t}+\varepsilon_{t}
$$

where $r_{M, t}$ is the excess market return for the entire sampling period (i.e., 2,519 observations ), and $\varepsilon_{t}$ and $r_{t}$ are a randomly generated error terms and stock returns. A value of zero for the alpha and one for the beta (i.e., $\alpha=0$ and $\beta=1$ ), are used in all random samples.

Following McDonald and White (1993), the regression errors are generated using the (1) normal, (2) mixed-normal (thick-tailed variancecontaminated), and (3) skewed log-normal distributions. Specifically, the normal error term is generated by $\varepsilon_{1}=\sigma z$, where $z \sim N(0,1)$. The thick-tailed variance contaminated error distribution is generated by $\varepsilon_{2}=$ $\sigma\left[w z_{1}+(1-w) z_{2}\right]$, where $z_{1} \sim N(0,1 / 9), z_{2} \sim N(0,9)$, and $w=1$ with probability 0.9 and $w=0$ with a probability of 0.1 . This distribution is symmetric and has a standardized kurtosis of 24.33. The log-normal distribution is generated by $\mathcal{E}_{3}=\sigma\left(e^{0.5 z}-e^{0.125}\right) /\left(e^{0.5}-e^{0.25}\right)^{0.5}$, where $z \sim$ $N(0,1)$. This distribution has standardized skewness 1.75 and standardized kurtosis of 8.898. The standard deviation $\sigma$ is computed using the equation $\sigma=\left[\left(1 / R^{2}\right)-1\right]^{0.5}|\beta| \sigma_{M}$, with $\beta=1, \sigma_{M}=1.1195$ (standard deviation of $r_{M, t}$ in the sample) so that the corresponding $R^{2}=$ 0.0879 is equal to the average CAPM $R^{2}$ for all stocks in the sample.

One thousand and fifty replications of samples are generated with the same three error distributions to estimate the alpha and beta parameters. Table 6 presents the means of the regression alphas for the normal, normal mixture, and log-normal samples and associated tstatistics in parentheses. Note that for the case of the normal and normal mixture random data the hypothesis of unbiased estimates of the 
regression intercept cannot be rejected at traditional levels of statistical significance for any of the estimators. In the case of log-normal (skewed) random data all of the symmetric models except OLS, i.e., the LAD, GED, student's T and GT provide biased estimates of the regression intercept. OLS estimators will be unbiased whenever the error terms have a zero mean and are uncorrelated with the regressors. They will be inefficient for non-normal error distributions such as those characterized by skewness or thick tails. The skewed models provide unbiased estimates of the regression intercepts. In the case of the slope, however, all models, symmetric and non-symmetric, provide unbiased estimates of the regression slopes.

Table 7 provides root mean squared errors (RMSE) of the nine estimation procedures in each of the three simulation samples for the intercept and slope estimators. The RMSE, computed as the square root of the sum of the sample variance of each estimator and the square of its sample bias, measures how close the estimator is to the true parameter.

Panel A of table 7, presents the results for the intercept estimator. In the case of the normal random sample, all models except LAD exhibit similar RMSE performance, thus there appears to be little efficiency loss for the intercept estimators relative to the OLS estimator. For the mixed normal random sample, the student's T and GT estimators are the best. The latter estimators are slightly better than the LAD and GED estimators. The remaining estimators are clearly inferior. For the lognormal random sample, OLS and all skewed estimators, including the ST and SGT, exhibit similar performance.

Panel B of table 7, presents the results for the slope estimator. In the case of the normal random sample, all slope estimators, except for those of LAD and SLAD, exhibit similar RMSE performance. For the mixednormal sample, the student's T, ST, GT and SGT slope estimators are the best. Their RMSE values are about $86 \%$ of those of GED and SGED, $71 \%$ of those of LAD and SLAD and $40 \%$ of that of OLS. Finally, in the case of the log-normal sample, the ST and SGT appear to be the best slope estimators, followed closely by the SGED estimator. The RMSE values of ST and GT are about $80 \%$ of those of SLAD and GT, $75 \%$ of that of student's T and about $63 \%$ of those of OLS, LAD and GED.

In sum, the simulation results for the intercept and slope estimators show that a) all models, except the LAD and SLAD, exhibit similar performances in the normal random sample, $b$ ) the student's T and GT are best estimators in the mixed-normal sample and c) the ST and SGT are the best estimators in the log-normal sample. Overall, the results favor the T and GT estimators in leptokurtic symmetric data and ST and 


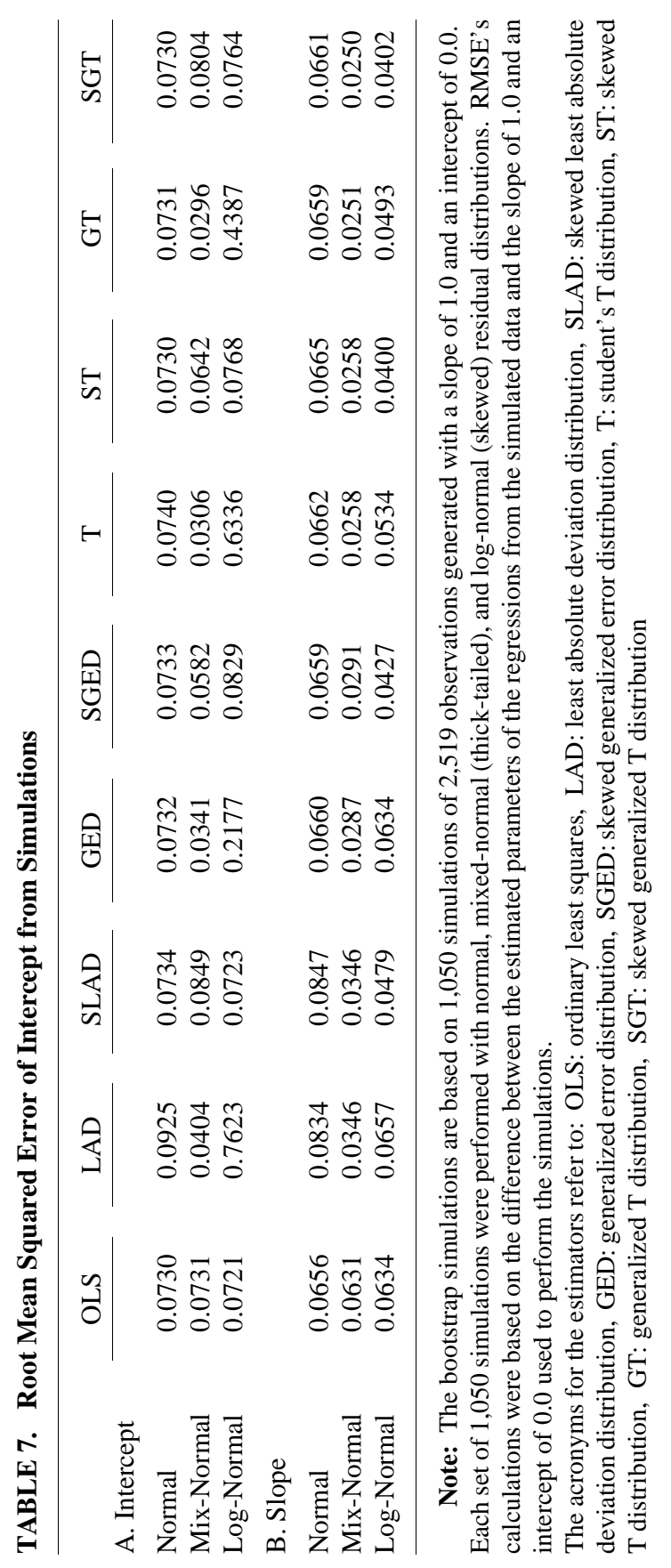


SGT estimators in skewed data. In normal samples, the OLS estimators are preferred because of their simplicity.

\section{Summary and Concluding Remarks}

This paper introduces a general class of quasi-maximum likelihood regression estimators based on the SGT distribution. This class of estimators includes the OLS, the LAD, the $L_{k}$, the trimmed regression quantile estimators, M-estimators, and the quasi-maximum likelihood estimators of symmetric and skewed student's T, Laplace, GED and GT probability distributions. As such, the SGT distribution provides a unified framework to investigate the impact of skewness on the estimated regression parameters of the various estimators and compare their relative efficiency in diverse types of data.

The importance and relevance of the various robust estimation techniques and impact of skewness on the estimated regression parameters is demonstrated using the CAPM, which involves regressing individual stock excess returns on market excess returns, and the entire universe of all publicly traded U.S. stocks with at least four years of data. A preliminary analysis of CAPM's regression residuals depict that about $97 \%$ of the stocks exhibit significant skewness and/or excess kurtosis, $79 \%$ of them exhibit significant positive skewness and $9 \%$ of them exhibit significant negative skewness. These results provide overwhelming support for the use of robust type estimation techniques for CAPM's estimation, and, more generally, any regression analysis of stock returns where the intercept is important. The empirical results include the impact of the occasional extreme error outlier as well as skewed errors.

Empirical and theoretical analysis shows that when skewness is present in the data, quasi-maximum likelihood estimation techniques based on symmetric probability distributions produce biased estimates for the regression intercepts. The latter bias is negative with negatively skewed data and positive with positively skewed data. The simulation results are generally consistent with the results of Newey and Steigerwald(1997) which show that a QMLE with a mis-specified symmetric error distribution provides an inconsistent estimator of the intercept when the true error distribution is asymmetric, but the QMLE can provide a consistent estimator of the intercept when the assumed error distribution is asymmetric and nests the true error distribution. The assumed error distribution, the SGT, does not include the lognormal as a special or limiting case, but allows the flexibility to capture 
the corresponding skewness and kurtosis. The latter has significant implications for finance, since the CAPM intercept, or Jensen's alpha, is frequently used in portfolio selection and stock and mutual fund valuation.

The simulation results using the normal, mixed-normal (thick-tails) and log-normal (positively skewed) random samples show that: a) all models, except the LAD and skewed LAD, exhibit similar performances in the normal random sample, b) the student's T and GT are the best estimators in the mixed-normal sample and c) the ST and SGT are the best estimators in the log-normal sample. Overall, the results favor the student's T and GT estimators in leptokurtic symmetric data and the ST and SGT estimators in skewed data. In normal samples, the OLS estimators are preferred because of their simplicity and to avoid efficiency loss from over-parameterization from having to estimate unnecessary distribution parameters.

The above findings are relevant and important to researchers in any area interested in unbiased and efficient regression estimators. Examples of skewed and/or leptokurtic data from other fields include a) building electricity usage data, e.g., Parti and Parti (1980), Hartman (1983), Bartels and Fiebig (1990); b) economic housing price data, e.g., Hansen, McDonald, and Turley (2006); c) meteorological solar radiation predictions and wind shear analysis data, e.g., Younes and Muneer (2006), Kanji (1985), Jones and McLachan (1990); and d) aeronautical flight navigation risk analysis, e.g., Hsu (1979).

Accepted by: Prof. R. Taffler, Guest Editor, November 2009

\section{Appendix A: Adjustment Factor and SGT Moments}

The sth non-centered moment of $u=r-\left(m+\beta r_{M}\right)$ for integer values of $s<n$ is

$$
\begin{aligned}
M_{s}= & C(-1)^{s} \int_{0}^{\infty} u^{s}\left(1+\frac{|u|^{k}}{((n+1) / k)(1-\lambda)^{k} \varphi^{k}}\right)^{-\left(\frac{n+1}{k}\right)} d u \\
& +C \int_{0}^{\infty} u^{s}\left(1+\frac{|u|^{k}}{((n+1) / k)(1+\lambda)^{k} \varphi^{k}}\right)^{-\left(\frac{n+1}{k}\right)} d u .
\end{aligned}
$$


Gradshteyn and Ryzhik (1994, p. 341) show that

$$
\int_{0}^{\infty} u^{s}\left(1+q u^{k}\right)^{-d} d u=k^{-1} q^{-(s+1) / k} B\left(\frac{d k-(s+1)}{k}, \frac{s+1}{k}\right)
$$

where $0<(s+1) / k<d, q \neq 0$ and $n \neq 0$.

Letting $q=((n+1) / k)(1-\lambda)^{-k} \varphi^{-k}$ or $q=((n+1) / k)(1+\lambda)^{-k} \varphi^{-k}$ and $d=(n+1) / k$ and substituting into the $M_{s}$ equation A.1 gives

$$
M_{s}=\left[(-1)^{s}(1-\lambda)^{s+1}+(1+\lambda)^{s+1}\right] C k^{-1}\left(\frac{n+1}{k}\right)^{\frac{s+1}{k}} B\left(\frac{n-s}{k}, \frac{s+1}{k}\right) \varphi^{s+1} .
$$

For $f($.$) to be proper probability density function,$

$$
\begin{gathered}
M_{0}=2 C k^{-1}\left(\frac{n+1}{k}\right)^{\frac{1}{k}} B\left(\frac{n}{k}, \frac{1}{k}\right) \varphi=1, \\
\text { thus } C=.5 k\left(\frac{n+1}{k}\right)^{-\left(\frac{1}{k}\right)} B\left(\frac{n}{k}, \frac{1}{k}\right)^{-1} \varphi^{-1} .
\end{gathered}
$$

Substitution of $C$ equation A.5 into the $M_{s}$ equation A.1 gives,

$$
\begin{aligned}
& M_{s}=0.5\left[(-1)^{s}(1-\lambda)^{s+1}+(1+\lambda)^{s+1}\right] \\
& B\left(\frac{n}{k}, \frac{1}{k}\right)^{-1}\left(\frac{n+1}{k}\right)^{\frac{s}{k}} B\left(\frac{n-s}{k}, \frac{s+1}{k}\right) \varphi^{s} .
\end{aligned}
$$

The expected value of $u$, provided that $n>1$, is

$$
E(u)=M_{1}=2 \lambda B\left(\frac{n}{k}, \frac{1}{k}\right)^{-1}\left(\frac{n+1}{k}\right)^{\frac{1}{k}} B\left(\frac{n-1}{k}, \frac{2}{k}\right) \varphi=\rho \varphi,(\mathrm{A} .7)
$$




$$
\text { where } \rho=2 \lambda B\left(\frac{n}{k}, \frac{1}{k}\right)^{-1}\left(\frac{n+1}{k}\right)^{\frac{1}{k}} B\left(\frac{n-1}{k}, \frac{2}{k}\right) \text {. }
$$

The second non-centered moment of $u$, provided that $n>2$, is

$$
\begin{gathered}
E\left(u^{2}\right)=M_{2}=0.5\left[(-1)^{2}(1-\lambda)^{3}+(1+\lambda)^{3}\right] \\
B\left(\frac{n}{k}, \frac{1}{k}\right)^{-1}\left(\frac{n+1}{k}\right)^{\frac{2}{k}} B\left(\frac{n-2}{k}, \frac{3}{k}\right) \varphi^{2} \\
=\left(1+3 \lambda^{2}\right) B\left(\frac{n}{k}, \frac{1}{k}\right)^{-1}\left(\frac{n+1}{k}\right)^{\frac{2}{k}} B\left(\frac{n-2}{k}, \frac{3}{k}\right) \varphi^{2}=\gamma \varphi^{2} .
\end{gathered}
$$

In this case the variance of $u$ is

$$
\sigma^{2}=E\left(u^{2}\right)-E(u)^{2}=\left(\gamma-\rho^{2}\right) \varphi^{2}
$$

where $\gamma-\tau^{2}>0$ (see below). In equation A.10 the variance, expressed in terms of $\varphi$, exists for as long as $n>2$, although the value of $\varphi$ exists for any value of $n>0$. Note that

$$
\begin{gathered}
\gamma-\tau^{2}=\left(1+3 \lambda^{2}\right) B\left(\frac{n}{k}, \frac{1}{k}\right)^{-1} B\left(\frac{n-2}{k}, \frac{3}{k}\right) \\
-4 \lambda^{2} B\left(\frac{n}{k}, \frac{1}{k}\right)^{-2} B\left(\frac{n-1}{k}, \frac{2}{k}\right)^{2} \\
=\left[1+3 \lambda^{2}-4 \lambda^{2} B\left(\frac{n}{k}, \frac{1}{k}\right)^{-1} B\left(\frac{n-2}{k}, \frac{3}{k}\right)^{-1} B\left(\frac{n-1}{k}, \frac{2}{k}\right)^{2}\right] \\
B\left(\frac{n}{k}, \frac{1}{k}\right)^{-1} B\left(\frac{n-2}{k}, \frac{3}{k}\right)^{-A}
\end{gathered}
$$




$$
=S(\lambda) B\left(\frac{n}{k}, \frac{1}{k}\right)^{-1} B\left(\frac{n-2}{k}, \frac{3}{k}\right)>0,
$$

because $S(\lambda)>0$ (the latter can be easily proven using the Stirling's approximation of the gamma function).

The third non-centered moment of $u$, provided that $n>3$, is

$$
\begin{gathered}
E\left(u^{3}\right)=M_{3}=0.5\left[(-1)^{3}(1-\lambda)^{4}+(1+\lambda)^{4}\right] \\
B\left(\frac{n}{k}, \frac{1}{k}\right)^{-1}\left(\frac{n+1}{k}\right)^{\frac{3}{k}} B\left(\frac{n-3}{k}, \frac{4}{k}\right) \varphi^{3} \\
=4 \lambda\left(1+\lambda^{2}\right) B\left(\frac{n}{k}, \frac{1}{k}\right)^{-1}\left(\frac{n+1}{k}\right)^{\frac{3}{k}} B\left(\frac{n-3}{k}, \frac{4}{k}\right) \varphi^{3}=A_{3} \varphi^{3} \text { (A.16) }
\end{gathered}
$$

The third center moment is,

$$
\begin{aligned}
& m_{3}=E\left(u-M_{1}\right)^{3}=E u^{3}-3 M_{1} E u^{2}+3 M_{1}^{2} E u-M_{1}^{3} \\
= & A_{3} \varphi^{3}-3 \gamma \rho \varphi^{3}+2 \rho^{3} \varphi^{3}=\left(A_{3}-3 \gamma \rho+2 \rho^{3}\right) \varphi^{3}=A_{3} \varphi^{3}
\end{aligned}
$$

The fourth non-centered moment of $u$, provided that $n>4$, is

$$
\begin{gathered}
E\left(u^{4}\right)=M_{3}=0.5\left[(-1)^{4}(1-\lambda)^{5}+(1+\lambda)^{5}\right] \\
B\left(\frac{n}{k}, \frac{1}{k}\right)^{-1}\left(\frac{n+1}{k}\right)^{\frac{4}{k}} B\left(\frac{n-4}{k}, \frac{5}{k}\right) \phi^{4} \\
=\left(1+10 \lambda^{2}+5 \lambda^{4}\right) B\left(\frac{n}{k}, \frac{1}{k}\right)^{-1}\left(\frac{n+1}{k}\right)^{\frac{4}{k}} B\left(\frac{n-4}{k}, \frac{5}{k}\right) \varphi^{4}=A_{4} \varphi^{4} .
\end{gathered}
$$


The fourth centered moment of $u$ is

$$
\begin{aligned}
m_{4}=E\left(u-M_{1}\right)^{4} & =E u^{4}-4 M_{1} E u^{3}+6 M_{1}^{2} E u^{2}-4 M_{1}^{3} E u+M_{1}^{4} \text { (A.21) } \\
& =\left(A_{4}-4 A_{3} \rho+6 \gamma \rho^{2}-3 \rho^{4}\right) \varphi^{4}
\end{aligned}
$$

The skewness and kurtosis measures are

$$
S K=\frac{m_{3}}{\sigma^{3}}=\frac{A_{3}-3 \gamma \rho+2 \rho^{3}}{\left(\gamma-\rho^{2}\right)^{3 / 2}}
$$

and

$$
K U=\frac{m_{4}}{\sigma^{4}}=\frac{A_{4}-4 A_{3} \rho+6 \gamma \rho^{2}-3 \rho^{4}}{\left(\gamma-\rho^{2}\right)^{2}}
$$

\section{Appendix B: Popular Distributions Nested by the SGT}

The skewed generalized T (SGT) distribution, developed by Theodossiou (1998),

$$
\begin{gathered}
f=.5 k\left(\frac{n+1}{k}\right)^{-\left(\frac{1}{k}\right)} B\left(\frac{n}{k}, \frac{1}{k}\right)^{-1} \\
\varphi^{-1}\left(1+\frac{|u|^{k}}{((n+1) / k)(1+\operatorname{sign}(u) \lambda)^{k} \varphi^{k}}\right)^{-\left(\frac{n+1}{k}\right)}
\end{gathered}
$$

"nests" several well known distributions.

For $\lambda=0$ it gives the generalized T (GT) of McDonald and Newey (1988)

$$
f=.5 k\left(\frac{n+1}{k}\right)^{-\left(\frac{1}{k}\right)} B\left(\frac{n}{k}, \frac{1}{k}\right)^{-1} \varphi^{-1}\left(1+\frac{|u|^{k}}{((n+1) / k) \varphi^{k}}\right)^{-\left(\frac{n+1}{k}\right)}
$$


McDonald (1989), McDonald and Newey (1988) and McDonald and Nelson (1989) used the GT to develop partially adaptive estimation of regression models. Butler et al. (1990) discussed the robust estimation of CAPM using the GT.

For $k=2$ it gives the Hansen's (1994) skewed T (ST)

$$
f=\left(\frac{n+1}{2}\right)^{-\left(\frac{1}{2}\right)} B\left(\frac{n}{2}, \frac{1}{2}\right)^{-1} \phi^{-1}\left(1+\frac{|u|^{2}}{((n+1) / 2)(1+\operatorname{sign}(u) \lambda)^{2} \varphi^{2}}\right)^{-\left(\frac{n+1}{2}\right)}
$$

used in autoregressive conditional density estimation.

For $k=2$ and $\lambda=0$ it gives the student's T distribution,

$$
f=\left(\frac{n+1}{2}\right)^{-\left(\frac{1}{2}\right)} B\left(\frac{n}{2}, \frac{1}{2}\right)^{-1} \phi^{-1}\left(1+\frac{|u|^{2}}{((n+1) / 2) \varphi^{2}}\right)^{-\left(\frac{n+1}{2}\right)}
$$

often used in log-likelihood specifications of data characterized by excess kurtosis, e.g., Bollerslev (1987).

For $n=1$ and $\lambda=0$ it gives the Cauchy distribution

$$
f=(\pi \phi)^{-1}\left(1+\frac{|u|^{2}}{\varphi^{2}}\right)^{-1}
$$

For $n=\infty$ it gives the skewed generalized error distribution (SGED) of Theodossiou (2001)

$$
f=0.5 k \Gamma\left(\frac{1}{k}\right)^{-1} \varphi^{-1} \exp \left(-\frac{|u|^{k}}{(1+\operatorname{sign}(u) \lambda)^{k} \varphi^{k}}\right),
$$

and for $\lambda=0$ the generalized error distribution (GED)

$$
f=0.5 k \Gamma\left(\frac{1}{k}\right)^{-1} \varphi^{-1} \exp \left(-\frac{|u|^{k}}{\varphi^{k}}\right) .
$$


The GED, introduced by Subbotin (1923), was used by Box and Tiao (1962) to model prior densities in Bayesian estimation, by Zeckhauser and Thompson (1970), Nelson (1991) and many others to model the distribution of financial return data.

For $k=2$ and $\varphi=\sqrt{2} \sigma$ and $\lambda=0$ the SGED gives the normal distribution.

$$
f=\frac{1}{\sqrt{2 \pi \sigma^{2}}} \exp \left(-\frac{|u|^{2}}{2 \sigma^{2}}\right)
$$

For $k=1$, it gives the skewed Laplace distribution

$$
f=0.5 \varphi^{-1} \exp \left(-\frac{|u|}{(1+\operatorname{sign}(u) \lambda) \varphi}\right)
$$

and for $k=1$ and $\lambda=0$ the Laplace distribution

$$
f=0.5 \varphi^{-1} \exp \left(-\frac{|u|}{\varphi}\right) .
$$

The Laplace distribution has found some very interesting applications. For example, Hsu (1979) used the Laplace to model the distribution of position errors in navigation, Kanji (1985) and Jones and McLachan (1990) to model the distribution of wind shear data and Bagchi, Hayya and Ord (1983) to model demand during lead and slow times.

Interestingly, maximum likelihood estimation using the GED, Laplace and skewed Laplace specifications yield some very well known estimators often used in regression estimation. Specifically, the GED log-likelihood specification, for a fix value of $k$, yields the $L_{k}$ estimator

$$
(\alpha, \beta)_{G E D}=\arg \left(\min _{\alpha, \beta} \sum_{t=1}^{T}\left|u_{t}\right|^{k}\right)
$$

Note that for $k=2$, equation B.11 gives the OLS estimator.

The Laplace log-likelihood specification yields the Lad or MAD estimator 


$$
(\alpha, \beta)_{\text {Laplace }}=\arg \left(\min _{\alpha, \beta} \sum_{t=1}^{T}\left|u_{t}\right|\right) \text {, }
$$

the skewed Laplace (SL) log-likelihood specification yields the (SLAD) the trimmed regression quantile (TRQ) estimator of Koenker and Bassett (1978); see also Chan and Lakonishok (1992),

$$
(\alpha, \beta)_{S L A D}=\arg \left(\min _{\alpha, \beta} \sum_{t=1}^{T} \rho\left(u_{t}\right)\right)
$$

where $\rho\left(u_{t}\right)=u_{t} /(1-\lambda)$ for $u_{t}<0$ and $\rho\left(u_{t}\right)=u_{t} /(1+\lambda)$ for $u_{t}>0$, where $-1<\lambda<1$; note in the trimmed regression quantile literature $1 /(1$ $-\lambda)=\theta$ and $1 /(1+\lambda)=1-\theta$, with $0<\theta<1$.

\section{References}

Bagchi, U.; Hayya, J.C.; and Ord, J.K. 1983. The Hermite Distribution as a Model of Demand During Lead-Time for Slow Moving Items, Decision Sciences, 14, 447-466.

Bartels, R., and Fiebig, D.J. 1990. Integrating Direct Metering and Conditional Demand Analysis for Estimating End-Use Load, The Energy Journal, 11, 79-97.

Blume, M. 1968. The Assessment of Portfolio Performance, Ph.D. Dissertation, University of Chicago.

Bollerslev, T. 1987. A Conditional Heteroskedastic Time Series Model for Speculative Prices and Rates of Return, Review of Economics and Statistics, 69, 542-547.

Box, G.E.P., and Tiao, G.C. 1962. A Further Look at Robustness Via Bayes Theorem. Biometrika, 49, 419-432.

Boyer, B.H.; McDonald, J.B.; and Newey, W.K. 2003. A Comparison of Partially Adaptive and Reweighted Least Squares Estimation, Econometric Reviews, 22, 115-134.

Butler, R.J.; McDonald, J.B.; Nelson, R.D.; and White, S. 1990. Robust and Partially Adaptive Estimation of Regression Models. Review of Economics and Statistics, 72, 321-327.

Chan, L.K.C., and Lakonishok, J. 1992. Robust Measurement of Beta. Journal of Financial and Quantitative Analysis, 27, 265-282.

Fama, E. 1965. The Behavior of Stock Market Movements. Journal of Business, 38, 1749-1778. 
Fama, E.; Fisher, L.; Jensen, M.; and Roll, R. 1969. The Adjustment of Stock Prices to New Information. International Economic Review, 10, 1-21.

Fielitz, B.D., and Smith, E.W. 1972. Asymmetric Distributions of Stock Price Changes. Journal of the American Statistical Association, 67, 813-814.

Francis, J.C. 1975. Skewness and Investor's Decisions. Journal of Financial And Quantitative Analysis, 10, 163-172.

Frankfurter, G.M.; Phillips, H.E.; and Seagle, J.P. 1974. Bias in Estimating Portfolio Alphas and Beta Scores. Review of Economics and Statistics, 56, 412-414.

Gradshteyn, I.S., and Ryzhik, I.M. 1994. Tables of Integrals, Series, and Products, $5^{\text {th }}$ ed., edited by Alan Jeffrey, New York: Academic Press.

Hampel, F.R. 1974. The Influence Curve and Its Role in Robust Estimation, Journal of the American Statistical Society, 69, 383-393.

Hansen, B.E. 1994. Autoregressive Conditional Density Estimation. International Economic Review, 35, 705-730.

Hansen, J.V.; McDonald, J.B.; and Turley, R.S. 2006. Partially Adaptive Robust Estimation of Regression Models and Applications, European Journal of Operations Research, 170, 132-143

Hansen, C.B.; McDonald, J.B.; and Theodossiou, P. 2007. Some Flexible Parametric Models for Skewed and Leptokurtic Data, Economics On-Line.

Hartman, R.S. 1983. The Estimation of Short-Run Household Electricity Demand Using Pooled Aggregate Data, Journal of Business and Economic Statistics, 1, 127-135.

Hinich, M.T., and Talwar., P.P. 1975. A Simple Method for Robust Regression. Journal of the American Statistical Association, 70, 113-119.

Hsu, D.A. 1979. Long-Tailed Distributions for Position Errors in Navigation, Applied Statistics, 28, 62-72.

Huber, P.J. 1981. Robust Statistics, New York: Wiley.

Jensen, M.C. 1968. The Performance of Mutual Funds in the Period 1945-1964. Journal of Finance, 23, 389-416.

Johnson, N. L. 1949. A Systems of Frequency Curves Generated by Methods of Translation, Biometrica, 36, 149-176.

Jones, P.N., and McLachlan, G.L. 1990. Laplace-Normal Mixtures Fitted to Wind Shear Data, Journal of Applied Statistics, 17, 283-291.

Kanji, G.K. 1985. A Mixture Model for Wind Shear Data, Journal of Applied Statistics, 12, 49-58.

Koenker, R. 1982. Robust Methods in Econometrics, Econometric Reviews, 1, 213-255.

Koenker, R., and Bassett, G. 1978. Asymptotic Theory of Least Absolute Regression Error. Journal of the American Statistical Association, 73, 618622.

Koenker, R., and Bassett, G. 1982. Robust Methods in Econometrics. (with discussion), Econometric Reviews, 1, 213-255.

Mandelbrot, B. 1963. The Variation of Certain Speculative Prices, Journal of 
Business, 36, 394-419.

Manski, C.F. 1984. Adaptive Estimation of Non-Linear Regression Models. Econometric Reviews, 3, 145-194.

Martin, R.G., and Simin, T.T. 2003. Outlier-Resistant Estimates of Beta, Financial Analysts Journal, 59, 56-69.

McDonald, J.B. 1989. Partially Adaptive Estimation of ARMA Models, International Journal of Forecasting, 5, 217-230.

McDonald, J.B.; Michelfelder, R.A.; and Theodossiou, P. 2009. Robust Estimators with Flexible Parametric Distributions: Estimation of Utility Stock Betas, forthcoming, Quantitative Finance.

McDonald, J.B., and Nelson, R.T. 1989. Alternative Beta Estimation for the Market Model using Partially Adaptive Estimation. Communications in Statistics: Theory and Methods, 18, 4039-4058.

McDonald, J.B., and Newey, W.K. 1988. Partially Adaptive Estimation of Regression Models Via the Generalized T Distribution. Econometric Theory, 4, 428-457.

McDonald, J.B., and White, S.B. 1993. A Comparison of Some Robust, Adaptive, and Partially Adaptive Estimators of Regression Models. Econometric Reviews, 12, 103-124.

McDonald, J.B., and Xu, Y.J. 1995. A Generalization of the Beta Distribution with Applications. Journal of Econometrics, 66, 133-152. Errata 69(1995), 427-428.

Nelson, D. 1991. A Conditional Heteroskedasticity in Asset Returns: A New Approach, Econometrica, 59, 347-370.

Newey, W.K., and Steigerwald, D.G. 1977. Asymptotic Bias for Quasi-Maximum-Likelihood in Conditional Heteroskedasticity Models. Econometrica, 65, 587-599.

Parti, M., and Parti, C. 1980. The Total and Appliance-Specific Conditional Demand for Electricity in the Household Sector, The Bell Journal of Economics, 11, 309-321.

Subbotin, M.T.H. 1923. On the Law of Frequency of Error. Mathematicheskii Sbornik, 31, 296-301.

Theodossiou, P. 1998. Financial Data and the Skewed Generalized T Distribution, Management Science, 44, 1650-1661.

Theodossiou, P. 2001. Skewness and Kurtosis in Financial Data and the Pricing of Options. Rutgers University.

Yohai, V.J., and Zamar, R.H. 1997. Optimal Locally Robust M-estimates of Regression, Journal of Statistical Planning and Inference, 64, 309-324.

Younes, S., and Muneer, T. 2006. Improvements in Solar Radiation Models Based on Cloud Data, Building Services and Engineering Research Technology Journal, 27, 41-54

Zeckhauser, R., and Thompson, M. 1970. Linear Regression with Nonnormal Error Terms, Review of Economics and Statistics, 52, 280-286. 